\title{
Acquired Hemophilia A with Gastrointestinal Bleeding
}

\author{
Narae Park, Jin Seok Jang and Jae Hwang Cha \\ Division of Gastroenterology, Department of Internal Medicine, Dong-A University College of Medicine, Busan, Korea
}

Peptic ulcer disease is the most common cause of acute gastrointestinal bleeding, followed by variceal bleeding, Mallory-Weiss syndrome, and malignancy. On the contrary, acquired hemophilia A is a very rare hemorrhagic disease, which usually manifests with musculocutaneous bleeding, caused by autoantibodies against coagulation factor VIII.

A 78-year-old man presented to the Emergency Department with melena. Dieulafoy's lesions were observed on esophagogastroduodenoscopy, and endoscopic cauterization was performed. However, the patient complained of back pain and symptoms indicative of upper gastrointestinal bleeding. Abdominopelvic computed tomography was performed, and hematoma in the psoas muscle was detected. Antibodies against coagulation factor VIII were confirmed with a blood test, and the diagnosis of acquired hemophilia A was made. Here, we report a case of acquired hemophilia A presenting with upper gastrointestinal bleeding symptoms and present a brief review of literature. Clin Endosc 2020;53:90-93

Key Words: Acquired; Factor VIII deficiency; Gastrointestinal hemorrhage; Hemophilia A

\section{INTRODUCTION}

The most common cause of acute upper gastrointestinal tract bleeding is peptic ulcer, and this may also occur due to variceal bleeding, Mallory-Weiss syndrome, or a malignant disease. ${ }^{1-3}$ However, upper gastrointestinal bleeding caused by acquired hemophilia A (AHA) has been reported very rarely worldwide. ${ }^{4}$ AHA is a rare disease that shows hemorrhagic tendencies due to autoimmune antibodies resistant to coagulation factor VIII (FVIII), and it occurs at a rate of $0.2-1$ per million people. ${ }^{5,6}$ The most common first symptom of AHA is hemorrhage in the skin and muscles. The mortality rate is $7.9 \%-22 \%$, and patients with such disease die within the first week after the first symptoms develop. ${ }^{7-9}$ We report a case and

Received: January 28, 2019 Revised: March 8, 2019

Accepted: March 13, 2019

Correspondence: Jin Seok Jang

Division of Gastroenterology, Department of Internal Medicine, Dong-A University College of Medicine, Dong-A University Hospital, 26 Daesingongwon-ro, Seo-gu, Busan 49201, Korea

Tel: +82-51-240-5042, Fax: +82-51-242-5852, E-mail: jsjang@dau.ac.kr ORCID: https://orcid.org/0000-0001-8067-4598

cc This is an Open Access article distributed under the terms of the Creative Commons Attribution Non-Commercial License (http://creativecommons.org/ licenses/by-nc/3.0) which permits unrestricted non-commercial use, distribution, and reproduction in any medium, provided the original work is properly cited. literature review of a patient with AHA who presented with upper gastrointestinal hemorrhage.

\section{CASE REPORT}

A 78-year-old man was admitted with a chief complaint of black stool. Ten days prior to his recent hospitalization, the patient was admitted to a nearby hospital with the same complaint. Esophagogastroduodenoscopy (EGD) was performed, and hemostatic procedures were done three times because of exudative hemorrhage and a Dieulafoy's lesion in the lesser curvature of the antrum and upper body of the stomach (Fig. 1). Melena was observed during digital rectal examination at the time of admission, and fresh red-colored blood was noted in gastric lavage via a nasogastric tube. The patient has been taking medications for hypertension, heart failure, and asthma, excluding anticoagulants or antiplatelet medications. He was a non-smoker and non-alcoholic.

At the time of visit, no symptoms other than the main complaint was noted during a systems review. Upon physical examination, he had an acute ill-looking appearance, and the conjunctiva and skin were slightly pale. There was no scleral jaundice and liver or spleen enlargement. His blood pressure 

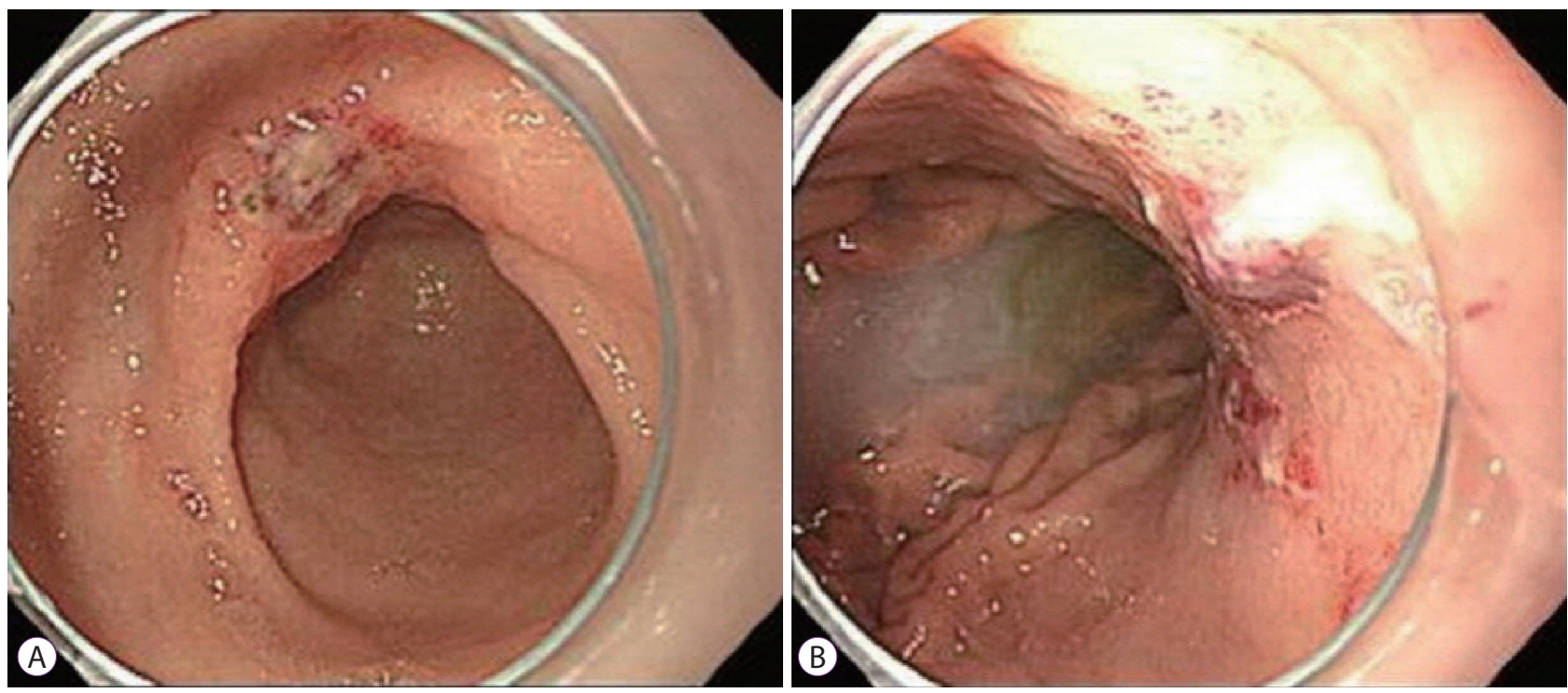

Fig. 1. Endoscopic findings: (A, B) Dieulafoy's lesions in the lesser curvature of the antrum and upper body of the stomach.

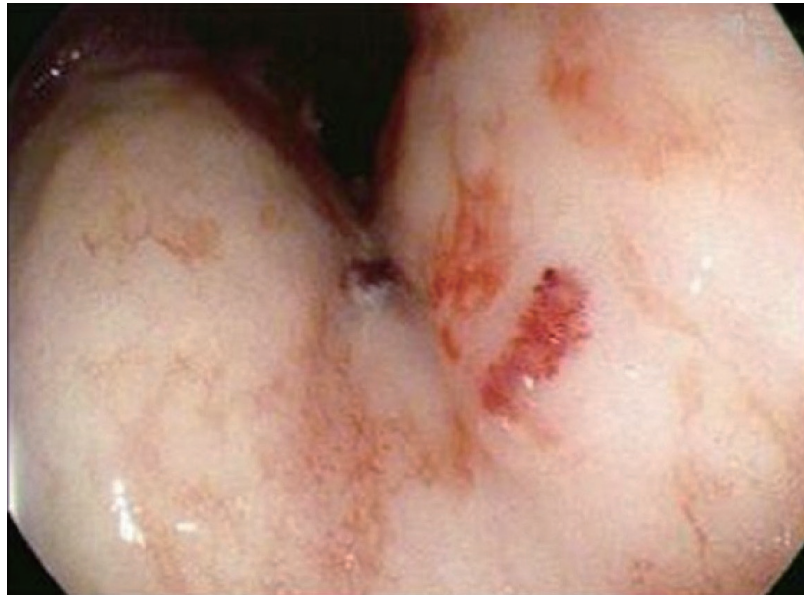

Fig. 2. Endoscopic findings: Angiodysplasia in the lesser curvature of the midbody of the stomach.

was 110/70 $\mathrm{mm} \mathrm{Hg}$, pulse rate was 98 times/min, respiratory rate was 20 times $/ \mathrm{min}$, and body temperature was $38.6^{\circ} \mathrm{C}$. Chest X-ray revealed no abnormalities, and the electrocardiogram showed sinus tachycardia.

Laboratory tests were conducted. Peripheral blood tests evaluated the leukocyte $\left(19,570 / \mathrm{mm}^{3}\right)$ and platelet count $\left(205,000 / \mathrm{mm}^{3}\right)$ and hemoglobin level $(5.8 \mathrm{~g} / \mathrm{dL})$. Biochemical tests included measurement of aspartate aminotransferase (17 $\mathrm{mg} / \mathrm{dL})$, alanine aminotransferase $(46 \mathrm{mg} / \mathrm{dL})$, total protein $(4.1 \mathrm{~g} / \mathrm{dL})$, albumin $(2.2 \mathrm{~g} / \mathrm{dL})$, total bilirubin $(1.0 \mathrm{mg} / \mathrm{dL})$, serum sodium $(135 \mathrm{mEq})$, and serum potassium $(3.7 \mathrm{mEq} / \mathrm{L})$ levels. Blood urea nitrogen $(41 \mathrm{mg} / \mathrm{dL})$, creatine $(1.24 \mathrm{mg} / \mathrm{dL})$, C-reactive protein $(11.89 \mathrm{mg} / \mathrm{dL})$ levels were all elevated. The prothrombin time (PT) was 15.4 seconds, and the activated

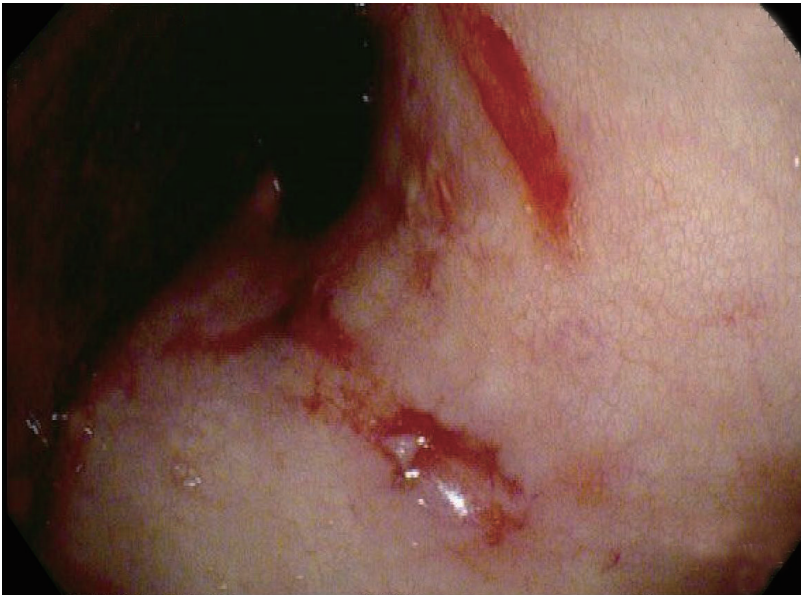

Fig. 3. Endoscopic findings: Oozing type bleeding in the lesser curvature of the upper body of the stomach.

partial thromboplastin time (aPTT) was 49.8 seconds, both of which were prolonged.

Since disseminated intravascular coagulation (DIC) due to repeated bleeding was suspected, the patient underwent an EGD based on the findings of melena and hemoglobin decline. Vascular formation was observed in the lesser curvature of the mid-body of the stomach, and argon plasma hemostasis was done (Fig. 2). On day 3 of admission, the hemoglobin level decreased, and follow-up EGD was performed. Argon plasma hemostasis revealed oozing bleeding in the upper body of the stomach (Fig. 3). The patient complained of back pain, and a bruise was observed on the right flank (Fig. 4). Computed tomography revealed a hematoma approximately $7 \mathrm{~cm}$ in size in the right psoas muscle (Fig. 5). On day 4 of admission, 


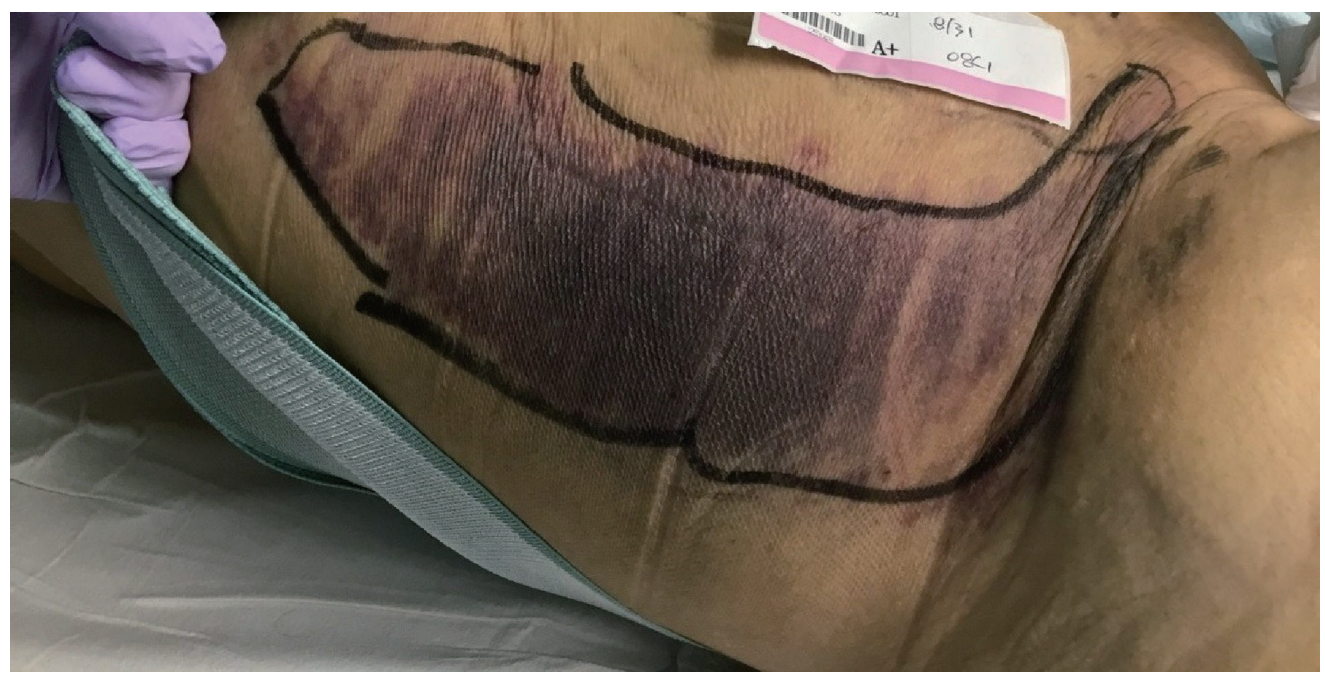

Fig. 4. Bruising in the right flank.

$650 \mathrm{cc}$ of melena was noted. Hence, EGD was performed. Results showed the presence of a gastric ulcer directly below the esophagogastric junction. An epinephrine solution was injected around the ulcer. On day 5, the patient complained of 200 cc of melena, and the hemoglobin level declined. Surgery was performed on the seventh day to achieve surgical hemostasis. At the time of surgery, there was no source of bleeding except on the area just below the esophageal junction. On the day following surgery, exudative hemorrhage from the surgical wound and $350 \mathrm{cc}$ of black stool were observed. Hemoglobin level was reduced to $6.0 \mathrm{~g} / \mathrm{dL}$, PT was prolonged to $14.9 \mathrm{sec}-$ onds, and aPTT was prolonged to 55.4 seconds. PT was corrected in the plasma mixing test; however, the aPTT was not corrected. In the additional test, antibody against blood coagulation FVIII (2.52 BU) was observed. Based on this test, the patient was diagnosed with AHA and bleeding. Then, he was transferred to the Hematology Department, and recombinant activated FVIII was administered. Consequently, the bleeding seemed to stop. Nonetheless, he refused recombinant activated FVIII because of the cost burden, and he died of shock on the 16th day of admission despite continued supportive care.

\section{DISCUSSION}

The cause of acute upper gastrointestinal bleeding varies, but the most common cause is peptic ulcer $(28 \%-59 \%)$. Non-steroidal anti-inflammatory drugs and Helicobacter $p y$ lori are independent risk factors for peptic ulcer and bleeding peptic ulcer. Additionally, chronic diseases such as cytomegalovirus, Crohn's disease, cirrhosis, chronic renal failure, and medications such as steroids, bisphosphonates, and fluorouracil can cause peptic ulcers. ${ }^{10,11}$ Despite endoscopic treatment, rebleeding occurred in $20 \%-22 \%$ in patients of peptic ulcer

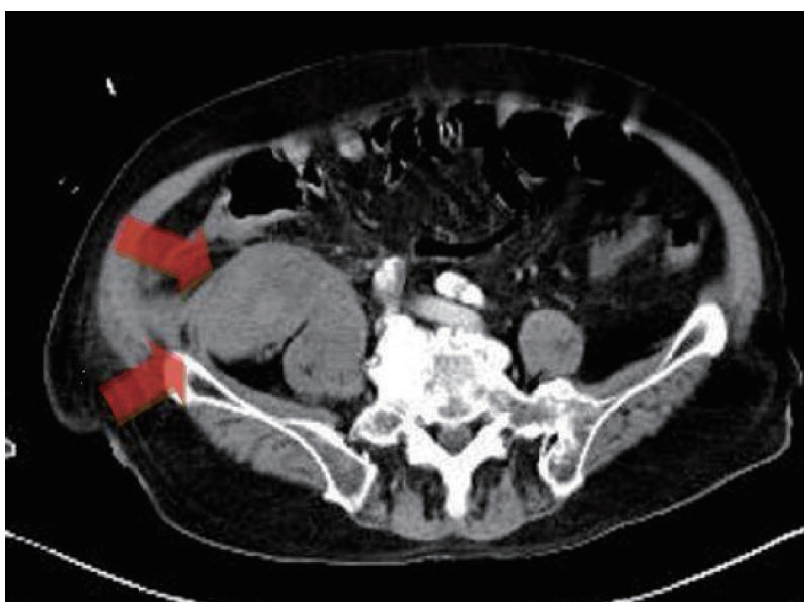

Fig. 5. A 7-cm hematoma in the right psoas muscle (arrow).

bleeding. ${ }^{12,13}$ The mortality rate of bleeding peptic ulcer was reported to be $2.4 \%-5.5 \%$, and the risk of death was higher among patients who were elderly and had hypertension, shock, and comorbidities. ${ }^{1,14,15}$ Moreover, upper gastrointestinal bleeding may occur due to variceal bleeding, Mallory-Weiss syndrome, and malignant disease. Upper gastrointestinal bleeding due to AHA has rarely been reported worldwide. ${ }^{2-4}$

AHA is a disease that has hemorrhagic tendencies caused by autoimmune antibodies resistant to coagulation FVIII, and it rarely occurs (0.2-1 per million people). ${ }^{5,6}$ It occurs idiopathically in more than half of the cases and is also associated with malignancy, autoimmune disorders, infection, and certain medications. ${ }^{5}$

The mortality rate is $7.9 \%-22 \%$, and the patients usually die within the first week after the appearance of the first symptom. ${ }^{7.8}$ The most common first symptom of AHA is hemorrhage in the skin and muscles. Spontaneously progressive posterior peritoneal hematoma or gastrointestinal bleeding is 
uncommonly reported. ${ }^{9}$ Clinical suspicion is raised because this is a very rare disease that may be suspected if prolongation of the aPTT without prolongation of PT is seen in patients with bleeding. ${ }^{16}$ If prolonged aPTT is normalized by a mixing test, hemophilia can be diagnosed. ${ }^{17}$ In patients with AHA, when bleeding occurs, recombinant FVIII or activated prothrombin complex concentrates may be administered; however, these may not be effective in patients with high antibody titers. ${ }^{5,18}$

The patient in this case report presented with a black stool. He had a history of hypertension but no history of medication use that could cause upper gastrointestinal bleeding. ${ }^{10,11}$ During an esophagoduodenoscopy, the common causes of upper gastrointestinal bleeding (peptic ulcer, varices, MalloryWeiss syndrome, and malignant diseases) were not observed. ${ }^{3}$ Unlike the usual upper gastrointestinal bleeding, bleeding occurred repeatedly despite endoscopic hemostasis. ${ }^{10}$ The patient underwent surgical treatment to manage the uncontrolled bleeding, and the bleeding focus was not clear at the time of surgery. PT and aPTT were prolonged simultaneously in the laboratory test. However, the aPTT was more prolonged than the PT, and the antibody to blood FVIII was confirmed. ${ }^{16}$ The first symptom in AHA is usually bleeding of the soft tissue; however, upper gastrointestinal bleeding is suspected to be the first manifestation of AHA in this patient. ${ }^{9}$

There was no history of liver disease and warfarin use, and repeated bleeding before admission was noted. Hence, the prolongation of PT and aPTT was considered to be caused by DIC. ${ }^{19}$ Thus, the diagnosis of AHA was delayed. Furthermore, for this reason, surgical treatment was performed to control hemorrhage, without requiring plasma exchange to reduce the antibodies before surgery. This was considered to be the cause of continued bleeding from the surgical wound. ${ }^{20}$ The patient was old; hence, no further treatment was conducted, and his prognosis was poor.

AHA is a very rare disease, and it is difficult to suspect it at first visit. ${ }^{5}$ However, considering hemophilia as an exclusive diagnosis in patients with sporadic hemorrhage and prolonged aPTT may increase the likelihood of diagnosis, avoid unnecessary treatments, and advance the appropriate treatment. Similarly, it may affect the patient prognosis. For this reason, we report this case of a patient with AHA due to upper gastrointestinal bleeding.

\section{Conflicts of Interest}

The authors have no financial conflicts of interest.

\section{REFERENCES}

1. Longstreth GF. Epidemiology of hospitalization for acute upper gastrointestinal hemorrhage: a population-based study. Am J Gastroenterol 1995;90:206-210.

2. Czernichow P, Hochain P, Nousbaum JB, et al. Epidemiology and course of acute upper gastro-intestinal haemorrhage in four French geographical areas. Eur J Gastroenterol Hepatol 2000;12:175-181.

3. van Leerdam ME. Epidemiology of acute upper gastrointestinal bleeding. Best Pract Res Clin Gastroenterol 2008;22:209-224.

4. McCain S, Gull S, Ahmad J, Carey D. Acquired hemophilia A as a cause of acute upper gastrointestinal hemorrhage. BMJ Case Rep 2014;2014.

5. Kruse-Jarres R, Kempton CL, Baudo F, et al. Acquired hemophilia A: updated review of evidence and treatment guidance. Am J Hematol 2017;92:695-705.

6. Oldenburg J, Zeitler H, Pavlova A. Genetic markers in acquired haemophilia. Haemophilia 2010;16 Suppl 3:41-45.

7. Bitting RL, Bent S, Li Y, Kohlwes J. The prognosis and treatment of acquired hemophilia: a systematic review and meta-analysis. Blood Coagul Fibrinolysis 2009;20:517-523.

8. Franchini M, Gandini G, Di Paolantonio T, Mariani G. Acquired hemophilia A: a concise review. Am J Hematol 2005;80:55-63.

9. Delgado J, Jimenez-Yuste V, Hernandez-Navarro F, Villar A. Acquired haemophilia: review and meta-analysis focused on therapy and prognostic factors. Br J Haematol 2003;121:21-35.

10. Ramakrishnan K, Salinas RC. Peptic ulcer disease. Am Fam Physician 2007;76:1005-1012.

11. Kurata JH, Nogawa AN. Meta-analysis of risk factors for peptic ulcer. Nonsteroidal antiinflammatory drugs, Helicobacter pylori, and smoking. J Clin Gastroenterol 1997;24:2-17.

12. Vreeburg EM, Snel P, de Bruijne JW, Bartelsman JF, Rauws EA, Tytgat GN. Acute upper gastrointestinal bleeding in the Amsterdam area: incidence, diagnosis, and clinical outcome. Am J Gastroenterol 1997;92:236243.

13. van Leerdam ME, Vreeburg EM, Rauws EA, et al. Acute upper GI bleeding: did anything change? Time trend analysis of incidence and outcome of acute upper GI bleeding between 1993/1994 and 2000. Am J Gastroenterol 2003;98:1494-1499.

14. Barkun A, Sabbah S, Enns R, et al. The Canadian registry on nonvariceal upper gastrointestinal bleeding and endoscopy (RUGBE): endoscopic hemostasis and proton pump inhibition are associated with improved outcomes in a real-life setting. Am J Gastroenterol 2004;99:1238-1246.

15. Blatchford O, Davidson LA, Murray WR, Blatchford M, Pell J. Acute upper gastrointestinal haemorrhage in west of Scotland: case ascertainment study. BMJ 1997;315:510-514.

16. Cohen AJ, Kessler CM. Acquired inhibitors. Baillieres Clin Haematol 1996;9:331-354.

17. Werwitzke S, Geisen U, Nowak-Göttl U, et al. Diagnostic and prognostic value of factor VIII binding antibodies in acquired hemophilia A: data from the GTH-AH 01/2010 study. J Thromb Haemost 2016;14:940-947.

18. Huth-Kühne A, Baudo F, Collins P, et al. International recommendations on the diagnosis and treatment of patients with acquired hemophilia $\mathrm{A}$. Haematologica 2009;94:566-575.

19. Toh C-H, Alhamdi Y, Abrams ST. Current pathological and laboratory considerations in the diagnosis of disseminated intravascular coagulation. Ann Lab Med 2016;36:505-512.

20. Ma AD, Carrizosa D. Acquired factor VIII inhibitors: pathophysiology and treatment. Hematology Am Soc Hematol Educ Program 2006:432437. 